\title{
Rabaska
}

Revue d'ethnologie de l'Amérique française

\section{PARADIS, ISABELLE. Les Maisons de l'Île m'ont raconté... Atelier découverte pour petits et grands curieux. [Sainte-Famille, Île d’Orléans], Fondation François-Lamy, 2018, 24 p.}

\section{René Bouchard}

Volume 17, 2019

URI : https://id.erudit.org/iderudit/1066038ar

DOI : https://doi.org/10.7202/1066038ar

Aller au sommaire du numéro

Éditeur(s)

Société québécoise d'ethnologie

ISSN

1703-7433 (imprimé)

1916-7350 (numérique)

Découvrir la revue

Citer ce compte rendu

Bouchard, R. (2019). Compte rendu de [PARADIS, ISABELLE. Les Maisons de l'île m'ont raconté... Atelier découverte pour petits et grands curieux. [Sainte-Famille, Île d'Orléans], Fondation François-Lamy, 2018, 24 p.] Rabaska, 17, 335-338. https://doi.org/10.7202/1066038ar d'utilisation que vous pouvez consulter en ligne. 
en question la croyance faussement et largement répandue que les Canadiens français n'avaient pas le sens des affaires ni l'esprit entrepreneurial. Elle ouvre la porte à des recherches sérieuses dans le domaine, elle invite à mieux documenter ces aspects négligés de notre histoire.

René Gagnon

Société d'histoire et de généalogie de la Côte-du-Sud

Paradis, Isabelle. Les Maisons de l'Île m'ont raconté... Atelier découverte pour petits et grands curieux. [Sainte-Famille, Île d'Orléans], Fondation François-Lamy, 2018, 24 p.

À l'heure où l'avenir du patrimoine bâti paraît plus sombre que jamais et suscite les plus vives inquiétudes devant le triste sort réservé à des icônes de l'architecture domestique et bourgeoise, comme à Lévis et Chambly de triste mémoire, et que l'idée s'ancre de plus en plus dans les esprits qu'un patrimoine qui s'appauvrit est un patrimoine qui dépérit avec notre identité, il est encourageant et réjouissant d'entendre des voix se lever et de voir des gestes posés pour changer les mentalités.

Isabelle Paradis, restauratrice spécialisée dans le traitement des œuvres en pierre au Centre de conservation du Québec (CCQ), est de cette classe. Passionnée par le patrimoine du Québec et celui de l'Île d'Orléans en particulier, elle a constitué une banque de données sur les maisons ancestrales au cours de ses travaux de restauration. Ces derniers l'ont amenée à approfondir ses recherches et à publier des articles précieux pour l'enrichissement de nos connaissances sur les maisons patrimoniales. Son expertise rayonne déjà auprès des architectes des quatre coins du Québec et on espère voir paraître bientôt son Guide sur la pierre et les mortiers, encore en chantier mais qui devrait bientôt enrichir une collection inaugurée par le CCQ avec la publication d'un premier guide technique sur Toit. Bois. Bardeau (Publications du Québec, 2016), devenu rapidement la référence par excellence pour les toitures de bois.

Entretemps, son analyse sur les finis architecturaux, parue dans Rabaska (vol. 16, 2018, p. 57-62), révélait déjà le caractère insoupçonné de l'étude des finis peints pour connaître plus en détail l'histoire des bâtiments anciens. «Encore mal connue en conservation architecturale au Québec, ce type d'étude, soutient la spécialiste, commence peu à peu à faire sa place dans les projets de restauration. " Autant « pour une maison historique, un bâtiment institutionnel ou une église, affirme-t-elle avec conviction, les couches stratigraphiques des finis peints permettent d'analyser les 
différents décors qui se sont succédé » et qui « donne[nt] accès, sous une forme condensée, à l'histoire du bâtiment ». Comme l'ont bien démontré les analyses comparatives des finis intérieurs de plusieurs habitations de l'Île d'Orléans avec la Maison Drouin, à Sainte-Famille, ce résultat a permis notamment de dresser un premier catalogue des couleurs de la maison rurale de l'Île entre 1690 et 1830 .

Ses connaissances terrain, l'auteure les a tirées également du rachat de la Maison Pichet-Gosselin, située à Saint-Laurent, dont elle a entrepris la restauration avec Pierre Laforest. Ce projet échelonné sur douze ans de patients et minutieux travaux a valu à ses auteurs le prix Thérèse-Romer 2013 de l'association des Amis et propriétaires de maisons anciennes du Québec (APMAQ), remis aux récipiendaires pour la conservation et la mise en valeur de leur maison ancestrale. Le journal de route de cette restauration exemplaire est rempli à ras bord de photos illustrant la complexité d'un tel chantier, marqué entre autres par la réfection de la maçonnerie de la fondation, le remplacement des poutres pourries en pièce sur pièce du carré initial et la réfection du parement extérieur en bois de la maison. Nous sommes ici, à l'évidence, dans le temps long de l'amour inconditionnel du patrimoine vivant...

Que, sur cette lancée, elle nous livre aujourd'hui un magnifique projet de médiation culturelle, un "Atelier découverte pour petits et grands curieux » des maisons de l'Île d'Orléans présentées comme des coffres aux trésors, accompagné d'une brochure destinée aux enfants, Les Maisons de l'Île m'ont raconté..., devrait en toute logique découler du pur plaisir de leur transmettre connaissances et passion pour le patrimoine architectural de leur coin de pays. La réalité est plus complexe qu'il n'y paraît de prime abord. Car parler patrimoine à l'Île, c'est souvent se faire l'écho des relations tendues entre les citoyens et le ministère de la Culture et des communications pour la délivrance des permis de travaux sur le seul site patrimonial en milieu rural au Québec. Plutôt que d'être pris entre l'arbre et l'écorce des récriminations de tout un chacun, pourquoi ne pas s'adresser à de jeunes esprits encore vierges et avides d'apprendre, avec l'espoir secret que ceux-ci deviennent auprès de leurs parents des vecteurs de connaissance, d'appartenance et de fierté ? C'est la voie suivie par l'auteure. « Les enfants, dira-t-elle, sont ouverts et curieux ; quand on les implique dans une activité, ils sont gourmands et généreux!»

Pour réaliser cette activité de participation culturelle, dont elle a accouché au bout de plus de neuf mois de gestation, elle a repris cette idée purement gratuite de procurer du plaisir aux enfants. "L'idée centrale de mon projet, dit-elle, c'est d'associer une grosse maquette de maison traditionnelle à la notion de jeu. De réaliser avec les enfants un atelier de 
construction miniature, un gros Lego du Régime français. Je voulais que les enfants touchent la maquette, qu'ils soient dans l'action plutôt que dans une sorte d'espace muséal peu propice à leurs ébats naturels. »

«Avec la maquette qu'ils construisent - ou déconstruisent, c'est selon-, les enfants ont le droit de frapper avec un marteau et je les encourage à taper fort, pour peu qu'ils en aient le goût. Par la manipulation des parties constitutives de la maquette d'une maison ancestrale, je leur donne beaucoup d'informations "sensibles" pour affiner leur regard sur les détails architecturaux. Je veux qu'ils gardent un souvenir positif de cette expérience, une petite graine d'amour planté dans leur cœur pour le patrimoine. Je n'insiste pas trop car je leur remets ma brochure à la fin de l'activité. J'espère secrètement qu'ils vont la parcourir attentivement, la chérir et la faire parvenir jusqu'à leurs parents pour que ceux-ci, à leur tour, trouvent le chemin conduisant à l'histoire intime de leur maison construite par leurs aïeux. »

La Fondation François-Lamy, qui a pour mission de préserver et mettre en valeur les lieux de mémoire orléanais, a saisi tout de suite la richesse de ce projet de médiation culturelle, ainsi que son fort potentiel patrimonial ; elle n'a pas hésité à soutenir sans réserve la publication de cette brochure qui offre une synthèse remarquable des connaissances accumulées sur les maisons de l'Île. Cette publication a été conçue pour des enfants ; elle comporte 24 pages et 61 illustrations qui démontrent l'importance accordée à l'image par rapport à l'écrit pour stimuler leur attention et les amener visuellement à capter l'essentiel de la matière. Quant à la maquette, cette dernière a été construite en érable, un bois avec lequel sont habituellement fabriqués les jouets d'enfant. Ses dimensions ( $\mathrm{H}: 91,5 \mathrm{~cm}$ x L : $122 \mathrm{~cm}$ x $\mathrm{P}: 91,5 \mathrm{~cm}$ ) ont été planifiées pour permettre aux enfants une manipulation aisée, et à l'auteure de la transporter sans problème, en pièces détachées, dans son coffre de voiture pour donner ses ateliers.

Rien n'est laissé au hasard dans le déroulement de l'histoire de la maison orléanaise racontée dans la brochure d'accompagnement, du paysage contemporain qu'elle embellit aux premiers balbutiements de son implantation au XVII siècle. Tout est passé en revue à ce chapitre au moyen de planches savamment illustrées et condensées. "Cabane » en pieux debout isolée à l'écorce de bouleau et maison en colombage pierroté héritée du Moyen Âge des débuts de la Nouvelle-France, jusqu'à la maison de bois en pièce sur pièce du XVIII ${ }^{\mathrm{e}}$ siècle, illustrent en même temps l'art et la façon de construire ces maisons avec des outils dont chacun avait une fonction précise. Scie de long, scie à refendre, scie à cadre, hache à équarrir, tarière, bédane, pour en nommer quelques-uns, servent aux divers assemblages du carré, à queue d'aronde ou à poteau à coulisse, comme aux différentes 
parties de la charpente. Cette dernière à son tour permet d'expliquer la forme du toit, tantôt à pavillon ou à croupe, tantôt à deux versants ou mansardé. À la maison de bois suit la maison de pierre de tradition française, sur le modèle des maisons de Normandie, du Perche ou de Bretagne. Massette, taillant, ciseaux et broche volent entre les mains des tailleurs de pierre pour marier savamment pierres et mortier. Quelques derniers coups d'œil sur les cheminées, vraies ou fausses, et sur l'allongement de l'avant-toit au XIX siècle font comprendre que les maisons de l'île constituent un patrimoine vivant, d'une valeur historique exceptionnelle. Voilà pourquoi elles font l'objet d'une réglementation particulière, explique l'auteure aux enfants, et pourquoi il faut les préserver au profit des générations futures. "Parce qu'il faut connaître pour aimer et aimer pour protéger », conclut l'auteure avec force en s'adressant à son public. Ironie du sort et pari réussi pour la restauratrice qui en avait fait un de ses objectifs les plus chers, ses nombreux ateliers, tenus en juin et juillet 2018 à la Maison Drouin, ont connu un grand succès tant auprès des petits que des grands curieux. Assurément des graines de vie en faveur du patrimoine qui ne tomberont pas sur le renchaussage !

S'il est par ailleurs un souhait que l'on pourrait adresser à l'auteure et à la Fondation François-Lamy en conclusion de la lecture de cette brochure méritoire, ce serait d'en confier la réalisation, dans la perspective d'une réimpression, à un professionnel de l'édition. Celui-ci verrait à corriger facilement les petites taches de naissance de l'œuvre, comme de lui rajouter une page des crédits en bonne et due forme, d'indiquer un numéro ISBN indispensable à la conservation de notre patrimoine imprimé, ou d'insérer une liste des figures et des sources qui s'y rapportent. Rien de bien majeur, mais un travail d'édition classique et indispensable pour parer à sa juste valeur un projet déjà remarquable de médiation culturelle très réussi.

RENÉ BouChaRd

Société québécoise d'ethnologie

Péruisset-Fache, Nicole. Civilisation, j'écris ton nom. Paris, L'Harmattan, 2018, 156 p. ISBN 978-2-343-14933-2.

Impossible de ne pas associer le titre de cet essai percutant à Liberté, le célébrissime poème que Paul Éluard écrivit en 1942 dans lequel « J'écris ton nom », scandé au dernier vers de chaque strophe, insuffle un rythme envoûtant, incantatoire, dont la progression ascensionnelle culmine sur " Liberté ». Au cœur de la Seconde Guerre mondiale, la liberté battait de l'aile. Éluard lui a imprimé cette pulsation émotionnelle afin qu'elle retrouve sa vitalité 